\title{
Factores de riesgo y prevalencia de trastornos migrañosos en población adulta. Estrategias médicas desde la atención primaria comunitaria
}

\author{
Risk factors and prevalence of migraine disorders in the adult population. Medical \\ strategies from community primary care
}

\author{
Elizabeth Salas Acosta1 ${ }^{1}$ Mireya Salas de González², Melissa González-Salas ${ }^{3}$
}

\begin{abstract}
RESUMEN
Objetivo: Determinar los factores de riesgo para trastornos migrañosos, presentes en población adulta hacia el establecimiento de estrategias médicas para su prevención y tratamiento desde la atención primaria comunitaria. Metodología: El estudio se desarrolló a través de una investigación tipo descriptivo y de campo, con una muestra de 64 pacientes, determinada a través de un muestreo no probabilístico intencional. Se diseñó un Cuestionario como instrumento de recolección de datos, cuya validación de contenido por medio de nueve expertos resultó suficiente. En el tratamiento estadístico se utilizó el programa Excel 2007 y SPSS versión 23, con un procedimiento tipo descriptivo, de frecuencias, sumatorias, media aritmética y porcentajes, representados en cuadros frecuenciales por indicadores. Resultados: Se demuestra prevalencia de antecedentes familiares en migraña, mientras que las características del dolor fue hemicraneal, pulsátil, intensidad moderada, insidioso, evolución con más de 5 años, siendo el naproxeno el medicamento más utilizado. Prevalencia de migraña sin aura en el sexo femenino entre las edades 31 a 60 años. El síntoma más experimentado además del dolor fue de vómitos/ nauseas. Conclusión: Se llega a la conclusión según los resultados que el mayor factor de riesgo desencadenante de la migraña fue de factores ambientales, traducido en la alta sensación térmica. Se recomienda la aplicación de lineamientos de estrategias locales en la promoción y prevención de salud.
\end{abstract}

Palabras clave: Factores de riesgo, trastornos migrañosos, atención primaria de salud.

\begin{abstract}
Objective: To determine the risk factors for migraine disorders, present in the adult population towards the establishment of medical strategies for their prevention and treatment from community primary care. Methodology: The study was developed through a descriptive and field type investigation, with a sample of 64 patients, determined through an intentional non-probabilistic sampling. A Questionnaire was designed as a data collection instrument, whose content validation by means of nine experts was sufficient. In the statistical treatment, Excel 2007 and SPSS version 23 were used, with a descriptive procedure of frequencies, summations, arithmetic mean and percentages, represented in frequency tables by indicators. Results: The prevalence of a family history of migraine is demonstrated, while the characteristics of the pain were hemicranial, throbbing, moderate intensity, insidious, evolution over 5 years, naproxen being the most widely used drug. Prevalence of migraine without aura in females between the ages of 31 to 60 years. The most experienced symptom besides pain was vomiting / nausea. Conclusion: According to the results, it is concluded that the greatest triggering risk factor for migraine was environmental factors, translated into high thermal sensation. The application of local strategy guidelines in health promotion and prevention is recommended.
\end{abstract}

Keywords: Risk factors, migraine disorders, primary health care.

\footnotetext{
${ }^{1}$ Universidad del Zulia, Zulia, Venezuela

Orcid ID: 0000-0003-0496-968X

${ }^{2}$ Centro de Investigaciones y desarrollo de Bioingeniería de la Costa Oriental del Lago (CIDIBICOL-UPTZ)

Orcid ID: 0000-0001-7740-8723

${ }^{3}$ Centro de Asesorias Educativas CEASE, Zulia, Venezuela

Orcid ID: 0000-0003-0686-1285
} 


\section{INTRODUCCIÓN}

Al estudiar diversidad de literatura científica sobre cefaleas y migraña, resulta coincidentes las aseveraciones realizadas por Vicente (2008) al declarar que "la migraña constituye un problema frecuente y también resulta ser uno de los primeros motivos de consulta en la atención primaria y asistencia neurológica especializada", por lo cual, se reconoce en el sistema de salud como un trastorno neurológico diferenciado.

Al describirla como un tipo de dolor de fuerte intensidad, los pacientes refieren que sienten pulsaciones en un área específica de la cabeza, asimismo presentan incomodidad ante la luz y el sonido, además de náuseas y vómitos en algunas oportunidades. Algunos pacientes refieren en las consultas que pueden sentir cuando van a tener migraña al ver destellos luminosos o perder temporalmente la vista antes de la aparición del dolor. Los trastornos migrañosos pueden perturbar la rutina diaria e interferir con el desempeño ocupacional, vida familiar o actividades recreativas, por ello se considera un problema de salud con implicaciones laborales, económicas y personales, pudiendo ser limitante en relación con la intensidad, duración y recurrencia. (Cady, 2014; Oterino \& Pascual, 2008; Titus, Fraga \& Martínez, 1992).

Realmente no se sabe con exactitud por qué se produce la migraña. Sin embargo, Pérez (2007) señala que la tendencia a padecerla puede ser hereditaria, corroborado por sus estudios que aseveran, si uno de los padres tiene migraña, existe un $40 \%$ de posibilidades de que su hijo las sufra. Si ambos padres tienen migraña, sus hijos tienen un $75 \%$ de posibilidades de padecerla también. Asimismo, manifiesta que en muchas mujeres el descenso del nivel de estrógeno que se produce inmediatamente antes de la menstruación, puede precipitar una migraña.

En consecuencia, diversos factores de riesgo pueden provocar su aparición, como algunos alimentos, medicamentos, estímulos sensoriales, cambios de hábitos, ciclo menstrual, cambios de estación o de presión atmosférica. En las personas sensibles, evitar estos desencadenantes, puede ayudar a reducir el grado de incapacidad; esto resulta importante en comunidades con situación económica contraída donde las posibilidades de adquisición de medicamentos y tratamientos médicos de alto costo son reducidas. Aunque existen diversos tratamientos farmacológicos, como el preventivo, de situaciones especial y tratamiento sintomático, también se plantea el tratamiento no farmacológico, como las acciones de promoción y prevención de salud que puede aprovechar el médico, para la educación del paciente acerca de su dolencia en una atención primaria de salud. En consecuencia, surge en primera instancia la idea de realizar este estudio considerando las recomendaciones de la Organización mundial de la Salud (OMS, 2008) en su informe anual del estado de salud a nivel global, sobre el fomento de estudios epidemiológicos, biomédicos y toxicológicos en Salud Ambiental para que el hombre tenga una vida digna y un medio adecuado.

Al respecto, la investigación plantea una problemática observada en la atención médica realizada en una comunidad del Estado Zulia, Venezuela donde un alto porcentaje de adultos dispensarizados presentan trastornos migrañosos como primordial patología junto a la Hipertensión arterial (HTA) y trastornos respiratorios. Por otro lado, es importante destacar el desconocimiento que tiene esta población sobre la información básica acerca de la migraña, evidenciado mediante entrevista no estructurada realizada durante la atención del paciente; por lo cual la investigación podría servir de base para formular programas de educación al respecto.

En tal sentido, es menester determinar científicamente, la epidemiología y etiología de la migraña en el sector señalado, evidenciando en la propia población los niveles de prevalencia de este trastorno de salud, así como las limitantes que ha representado esta dificultad en su propio organismo y desenvolvimiento social. De tal manera que el objetivo general de la investigación fue determinar los factores de riesgo para trastornos migrañosos en una población adulta del Estado Zulia, Venezuela, para consecuentemente diseñar lineamientos estratégicos de promoción y prevención de salud referidos a evitar la evolución de los episodios de migraña.

\section{METODOLOGÍA}

A través de una investigación descriptiva con diseño de campo, no experimental, de corte transversal orientada a recolectar informaciones desde el estado real y en el espacio donde ocurren los hechos para describirlos e interpretar su naturaleza (Hernández, et al. 2010; Chávez, 
2003), se estudia durante un año, una muestra de 64 pacientes adultos dispensarizados con presunto diagnóstico de migraña. El muestreo empleado fue no probabilístico intencional (Padua, 2002), sobre la población de 1.694 pacientes del consultorio popular Salud y Vida, situado en una comunidad de bajos recursos económicos en el Estado Zulia, Venezuela; acorde a los siguientes criterios de inclusión, señalados por Pascual (2012) y Silberstein (2004): que fueran pacientes mayores de 18 años, de ambos sexos, consultas reiteradas, presentaran al menos tres síntomas asociados a la migraña. De esta forma, se ubican los sujetos seleccionados como muestra, se citan a consulta en el Modulo de salud, y se incluyen definitivamente en el estudio una vez que autorizan su participación en la investigación, mediante la firma de un comunicado pidiendo su colaboración al respecto.

Como instrumento de recolección de datos se emplea un cuestionario tipo historial médico, validado por nueve especialistas, divido en 4 secciones referidas a: Abordaje diagnóstico, Prevalencia, Síntomas asociados a la migraña y Factores de riesgo. Las preguntas y proposiciones fueron diseñadas de forma cerrada, en escala dicotómica o de selección cerrada.
A la información recolectada se le aplica tratamiento estadístico descriptivo, procesado en Microsoft Excel 2007 y el software SPSS versión 23, determinando frecuencias, sumatorias, media aritmética y porcentajes, representados en cuadros frecuenciales por indicadores y dimensiones. Cabe destacar que este estudio fue revisado y aprobado por el comité de ética conformado por médicos docentes del Instituto de Altos Estudios Dr Arnoldo Gabaldón y de la Universidad del Zulia, Venezuela.

\section{RESULTADOS}

El abordaje diagnóstico de la migraña, inicia con la Anamnesis de los antecedentes personales y familiares en la Tabla 1, con media aritmética de $79 \%$ de los pacientes sin antecedentes personales y resaltando $88 \%$ con antecedentes familiares en primer grado. Los antecedentes personales, con mayor porcentaje de prevalencia son los Trastornos de la refracción ocular en $64 \%$, sinusitis en $56 \%$ y $53 \%$ con patologías dentales, cada uno de los cuales pudiera estar ocasionando las crisis de migraña presentada.

Tabla 1

Anamnesis. Antecedentes personales y familiares

\begin{tabular}{|c|c|c|c|c|}
\hline \multirow{2}{*}{ Antecedentes personales } & \multicolumn{2}{|c|}{ SI } & \multicolumn{2}{|c|}{ NO } \\
\hline & $\mathrm{fi}$ & $\%$ & fi & $\%$ \\
\hline Sinusitis & 36 & 56 & 28 & 44 \\
\hline Otomastoiditis & 01 & 02 & 63 & 98 \\
\hline Traumatismo craneoencefálico & 0 & 0 & 64 & 100 \\
\hline Cervicoartrosis & 06 & 09 & 58 & 91 \\
\hline Patología dental & 34 & 53 & 30 & 47 \\
\hline Patología de la articulación temporomandibular & 0 & 0 & 64 & 100 \\
\hline Glaucoma & 0 & 0 & 64 & 100 \\
\hline Trastornos de la refracción ocular & 41 & 64 & 23 & 36 \\
\hline Punción lumbar reciente & 01 & 02 & 63 & 98 \\
\hline$\Sigma$ & 119 & 186 & 457 & 714 \\
\hline $\bar{x}$ & 21 & & 79 & \\
\hline Antecedentes familiares de migraña & 56 & 88 & 08 & 12 \\
\hline
\end{tabular}

Fuente: Elaboración propia

En la anamnesis dirigida a la cefalea, la tabla 2 presenta $80 \%$ con dolor localizado hemicraneal; $13 \%$ holocraneal, $0 \%$ en banda; a nivel fronto-orbitaria se presenta al $4 \%$ y occipital a $3 \%$ de los sujetos. En la localización del dolor migrañoso, $80 \%$ presentan de carácter hemicraneal, Mientras que en las 
características de la cefalea, predomina en $70 \%$ carácter pulsátil cuando su intensidad aumenta; $28 \%$ la caracterizan como opresiva y $2 \%$ punzante. De intensidad moderada en un $64 \%$ y $36 \%$ de forma intensa. Ninguno reportó intensidad leve, ni incapacitante.

Tabla 2

Anamnesis dirigida a la cefalea

\begin{tabular}{|c|c|c|}
\hline Sección & $\mathrm{fi}$ & $\%$ \\
\hline Localización del dolor & " & 10 \\
\hline Occipital & 2 & 3 \\
\hline Fronto-orbitaria & 3 & 4 \\
\hline En banda & 0 & 0 \\
\hline Holocraneal & 8 & 13 \\
\hline Hemicraneal & 51 & 80 \\
\hline Características de la cefalea & fi & $\%$ \\
\hline Explosiva & 0 & 0 \\
\hline Un peso & 0 & 0 \\
\hline Tirante & 0 & 0 \\
\hline Punzante & 1 & 2 \\
\hline Opresiva & 18 & 28 \\
\hline Pulsátil & 45 & 70 \\
\hline Intensidad & $\mathrm{fi}$ & $\%$ \\
\hline Incapacitante & 0 & 0 \\
\hline Intensa & 23 & 36 \\
\hline Moderada & 41 & 64 \\
\hline Leve & 0 & 0 \\
\hline Modo de instauración & fi & $\%$ \\
\hline Insidioso & 38 & 59 \\
\hline En minutos & 25 & 39 \\
\hline Brusco & 1 & 2 \\
\hline Tiempo de evolución & $\mathrm{fi}$ & $\%$ \\
\hline Más de 5 años & 49 & 76 \\
\hline Meses & 14 & 22 \\
\hline Semanas & 1 & 2 \\
\hline Duración del episodio de cefalea & $\mathrm{fi}$ & $\%$ \\
\hline Varios días & 41 & 64 \\
\hline Pocas horas & 23 & 36 \\
\hline Frecuencia aproximada & fi & $\%$ \\
\hline Cada mes & 38 & 59 \\
\hline Cada 15 días & 25 & 39 \\
\hline 1 semana & 1 & 2 \\
\hline Medicación & $\mathrm{fi}$ & $\%$ \\
\hline Ibuprofeno & 16 & 25 \\
\hline Naproxeno & 30 & 47 \\
\hline Aspirina & 1 & 2 \\
\hline Paracetamol & 9 & 14 \\
\hline Otros & 8 & 12 \\
\hline
\end{tabular}

Fuente: Resultados de la búsqueda. 
En el modo de instauración es mayormente insidioso, corroborado por $59 \%$ de pacientes; $39 \%$ manifiesta la instauración en minutos y $2 \%$ de forma brusca. Asimismo, $76 \%$ presentan un tiempo de evolución de más de 5 años; $22 \%$ en meses y $2 \%$ en semanas. Asimismo, la duración de los episodios de cefalea mayormente se reporta de varios días, afirmado por $64 \%$, mientras que $36 \%$ presentan episodios de pocas horas. La frecuencia aproximada de la cefalea, puede observarse en $59 \%$ cada mes; $39 \%$ cada 15 días y $2 \%$ cada semana. Respecto a la medicación se muestra que $47 \%$ utilizan naproxeno, $25 \%$ ibupofreno; $14 \%$ paracetamol, $2 \%$ aspirina y $12 \%$ otros medicamentos que generalmente son esteroides.

En la exploración física general, evaluada en la Tabla 3, se determina que la auscultación craneal resultó normal en el $100 \%$ de los sujetos, igualmente la Palpación de los senos paranasales y articulación temporomandibular se reflejó como normal 100\%, sin embargo, en la palpación de cadenas ganglionares, resultó palpable en $6 \%$ mientras que en $94 \%$ fue normal. Por otra parte, la palpación del trago auditivo y mastoides y la palpación de las apófisis espinosas cervicales y musculatura cervical, así como palpación abdominal estuvo dentro de los límites normales en $100 \%$ en cada caso; no así en la auscultación cardiopulmonar reportada en $22 \%$ con HTA y $78 \%$ considerado normal.

Tabla 3

Exploración física general

\begin{tabular}{lllll} 
Exploración & Normal & \multicolumn{3}{c}{ Anormal } \\
\cline { 2 - 5 } Auscultación craneal & $\mathrm{fi}$ & $\%$ & $\mathrm{fi}$ & $\%$ \\
Palpación de los senos paranasales y articulación temporomandibular & 64 & 100 & 0 & 0 \\
Palpación de cadenas ganglionares & 64 & 100 & 0 & 0 \\
Palpación del trago auditivo y mastoides & 60 & 94 & 04 & 06 \\
Palpación de las apófisis espinosas cervicales y musculatura cervical & 64 & 100 & 0 & 0 \\
Auscultación cardiopulmonar & 64 & 100 & 0 & 0 \\
Palpación abdominal & 50 & 78 & 14 & 22 \\
\hline
\end{tabular}

Fuente: Elaboración propia

En la exploración neurológica, la Tabla 4 presenta aspectos normales en su totalidad: Nivel de conciencia, orientación y funciones cerebrales superiores; pares craneales y función pupilar; balance muscular, sensibilidad, reflejos osteotendinosos y cutaneoplantares, coordinación, marcha y equilibrio. La presencia de signos de irrigación meníngea es negativa: Rigidez de nuca; signo de Brudzinski; signo de Kerning.

Tabla 4

Exploración neurológica

\begin{tabular}{lllll} 
Exploración & Normal & Anormal \\
\cline { 2 - 5 } Nivel de conciencia, orientación y funciones cerebrales superiores & $\mathrm{fi}$ & $\%$ & $\mathrm{fi}$ & $\%$ \\
Pares craneales y función pupilar & 64 & 100 & 0 & 0 \\
$\begin{array}{l}\text { Balance muscular, sensibilidad, reflejos osteotendinosos y cutaneoplantares, coordi- } \\
\text { nación, marcha y equilibrio }\end{array}$ & 64 & 100 & 0 & 0 \\
\hline & 100 & 0 & 0 \\
\hline Signos de irrigación meníngea: Rigidez de nuca & Negativo & Positivo \\
Signos de irrigación meníngea: Signo de Brudzinski & 64 & 100 & 0 & 0 \\
Signos de irrigación meníngea: Signo de Kerning & 64 & 100 & 0 & 0 \\
\hline
\end{tabular}

Fuente: Elaboración propia 
Finalmente, se presentan en la Tabla 5, los resultados de las pruebas complementarias, que deben incluirse en el abordaje diagnóstico cuando existe sospecha de una etiología orgánica extracraneal de la cefalea, determinando que $100 \%$ obtuvo resultado negativo en los exámenes de laboratorio, radiología simple craneal y radiología cervical. En los estudios de neuroimagen, la tomografía computarizada y resonancia magnética practicada a aquellos pacientes que lo requerían, resultó $100 \%$ negativas. En el caso de migraña con auras atípicas o de larga duración los estudios de hipercoagulabilidad fueron $100 \%$ negativos. Como complemento se diagnosticó que la situación personal, laboral y familiar en el aspecto psicológico predisponente al estrés y consecuente migraña fue $52 \%$ favorable y $48 \%$ desfavorable según datos suministrados por los sujetos.

Tabla 5

Pruebas complementarias

\begin{tabular}{|c|c|c|c|c|}
\hline \multirow{2}{*}{ En sospecha de etiología orgánica extracraneal de la cefalea } & \multicolumn{2}{|c|}{ Negativo } & \multicolumn{2}{|c|}{ Positivo } \\
\hline & $\overline{\mathrm{fi}}$ & $\%$ & $\mathrm{fi}$ & $\%$ \\
\hline Laboratorio & 64 & 100 & 0 & 0 \\
\hline Radiología simple craneal & 64 & 100 & 0 & 0 \\
\hline Radiología cervical & 64 & 100 & 0 & 0 \\
\hline \multicolumn{5}{|l|}{ Estudios de neuroimagen } \\
\hline Tomografía Computarizada con contraste & 64 & 100 & 0 & 0 \\
\hline Resonancia Magnética & 64 & 100 & 0 & 0 \\
\hline \multicolumn{5}{|l|}{ En migraña con auras atípicas o de larga duración } \\
\hline Estudio de hipercoagulabilidad & 64 & 100 & 0 & 0 \\
\hline Complemento psicológico de estrés & \multicolumn{2}{|c|}{ Favorable } & \multicolumn{2}{|c|}{ Desfavorable } \\
\hline Situación personal, laboral y familiar & 33 & 52 & 31 & 48 \\
\hline
\end{tabular}

En la dimensión referida a la prevalencia de la migraña, la Tabla 6 presenta los tipos de migraña en los pacientes por edad y sexo; específicamente $48 \%$ padeciendo de migraña sin aura, de los cuales $39 \%$ son del sexo femenino y $09 \%$ del sexo masculino. De estos datos se evidencia que $16 \%$ son de edades comprendidas entre 18 a 30 años de edad, $31 \%$ son de 31 a 60 años y $2 \%$ de 61 y más. La migraña con aura es presentada por $44 \%$; $38 \%$ mujeres y $6 \%$ hombres. De ellos, $30 \%$ son de 31 a 60 años y $14 \%$ son de 18 a 30 años. En la migraña vertebrobasilar no se presentó ningún caso; mientras que en la migraña transformada por abuso de analgésico se presenta $8 \%$ de casos, en su totalidad son de sexo femenino y entre 31 a 60 años. La sumatoria en la Tabla 6, indica que $85 \%$ son de sexo femenino y $15 \%$ masculino; $69 \%$ en edad de 31 a $60,30 \%$ de 18 a 30 y $2 \%$ de 61 y más.

Tabla 6

Prevalencia por tipo de migraña, según edad y sexo

\begin{tabular}{|c|c|c|c|c|c|c|c|c|c|c|c|c|}
\hline \multirow{3}{*}{ Tipo de migraña } & \multicolumn{2}{|c|}{ Migraña } & \multicolumn{6}{|c|}{ Edad } & \multicolumn{4}{|c|}{ Sexo } \\
\hline & \multirow[t]{2}{*}{$\mathrm{fi}$} & \multirow[t]{2}{*}{$\%$} & \multicolumn{2}{|c|}{18 a 30} & \multicolumn{2}{|c|}{31 a 60} & \multicolumn{2}{|c|}{$\begin{array}{l}61 \mathrm{y} \\
\text { mas }\end{array}$} & \multicolumn{2}{|l|}{ M } & \multicolumn{2}{|l|}{$\mathbf{F}$} \\
\hline & & & $f i$ & $\%$ & $f i$ & $\%$ & $f i$ & $\%$ & $\mathrm{fi}$ & $\%$ & fi & $\%$ \\
\hline Migraña sin aura & 31 & 48 & 10 & 16 & 20 & 31 & 01 & 2 & 06 & 09 & 25 & 39 \\
\hline Migraña con aura & 28 & 44 & 09 & 14 & 19 & 30 & 0 & 0 & 04 & 06 & 24 & 38 \\
\hline Migraña vertebrobasilar & 0 & 0 & 0 & 0 & 0 & 0 & 0 & 0 & 0 & 0 & 0 & 0 \\
\hline $\begin{array}{l}\text { Migraña transformada con abuso } \\
\text { de analgésicos. }\end{array}$ & 05 & 08 & 0 & 0 & 05 & 08 & 0 & 0 & 0 & 0 & 05 & 08 \\
\hline$\Sigma$ & 64 & 100 & 19 & 30 & 44 & 69 & 1 & 2 & 10 & 15 & 54 & 85 \\
\hline
\end{tabular}

Fuente: Elaboración propia 
Se aclara que el caso de la dimensión síntomas asociados a la migraña, por registrarse más de un dato por cada paciente, se analiza estadísticamente desde la frecuencia y no del porcentaje en sí; ya que los pacientes pueden presentar varios síntomas a la vez. La tabla 7 presenta 62 pacientes con síntoma más frecuente, dolor entre moderado a intenso; 59 un dolor palpitante; 47 Nauseas/vómitos; 40 pacientes refieren foto-sonofobia; 36 presentan auras y 2 reportan pérdida de una parte del campo visual. Resulta importante reseñar que la descripción de los síntomas más frecuentes presentados, es significativo ya que el dolor puede venir acompañado con mayor o menor frecuencia de otros síntomas, cuya aparición es determinante en algunos casos para el diagnóstico diferencial.

Tabla 7

Síntomas más frecuentes asociados a la migraña

\begin{tabular}{lll} 
Síntoma & $\mathrm{fi}$ & $\%$ \\
\hline Aurea: Fenómenos visuales & 36 & 56 \\
Dolor entre moderado e intenso & 62 & 97 \\
Dolor palpitante & 59 & 92 \\
Pérdida de una parte del campo visual & 02 & 03 \\
Foto-sonofobia & 40 & 63 \\
\hline Náuseas/Vómitos & 47 & 73 \\
\hline
\end{tabular}

Fuente: Elaboración propia

Todos los planteamientos de los resultados referidos hasta el momento, sirvieron de antesala para concretar los Factores de riesgo en la tabla 8, motivo principal de la investigación. Los factores de riesgos, también presentaron respuestas simultáneas en un mismo caso, se observa que 63 pacientes consideran factores ambientales con mayor tendencia a la cronicidad de la migraña. Seguidamente se presenta el factor estímulos sensoriales en 60 pacientes, constituyéndose también como un componente considerable. Además, se observa el Cambio de hábitos en 56 personas; algunos alimentos en 46; medicamentos en 3 y Factores genéticos en 2 sujetos.

Tabla 8

Factores moduladores del dolor

\begin{tabular}{lll} 
Síntoma & fi & \% \\
Alimentos & 46 & 72 \\
Medicamentos & 03 & 95 \\
Estímulos sensoriales & 60 & 56 \\
Cambio de hábitos & 56 & 02 \\
Factores genéticos & 63 \\
Factores ambiental & 63 \\
\hline
\end{tabular}

Fuente: Elaboración propia

\section{DISCUSIÓN}

En los resultados del abordaje diagnóstico de la migraña, los altos porcentajes de pacientes con antecedentes familiares corroboran la afirmación de las investigaciones de Oterino y Pascual (2008), Terwindt et al. (1998) y McCandless et al (2011) sobre la gran cantidad de sujetos migrañosos que tienen familiares de primer grado con migraña, y antecedentes de otras enfermedades congénitas que pudieran relacionarse con este trastorno.
La localización del dolor hemicraneal permitió clasificarlo como uno de los más frecuentes, sin embargo, tal como lo señala Ezpeleta (2007); quien coincide en resultados similares en algunos de sus estudios; no obstante considera necesario seguir indagando siempre en un diagnóstico diferencial acerca de las características, intensidad, evolución, entre otros, para determinar con precisión el tipo de migraña. 
Respecto a las características, prevalece el carácter pulsátil con una intensidad moderada resaltando según los antecedentes de estudio de Vicente (2008), que la intensidad tiene un valor diagnóstico menor, pero debe precisarse en una escala de 1 a 10; para seguir la evolución del paciente. La intensidad de la cefalea puede determinarse según el impacto que produzca en las actividades habituales del paciente. Será leve si no altera las actividades; mediana si las dificulta parcialmente y severa si las impide. Para la muestra en cuestión, en su mayoría, consideran la migraña moderada como una patología que le obstaculiza objetivamente llevar a cabo su trabajo. Se registró un modo de instauración mayormente insidioso, con un tiempo de evolución prevalente de más de 5 años. Según su duración, se caracteriza por episodios recurrentes en los que la cefalea puede durar entre unas horas a varios días, coincidiendo con los estudios de Uribe (2008) donde se encontró de manera similar que $70 \%$ de las mujeres presentan duración de los ataques de dolor de cabeza a más de 24 horas y el $48 \%$ de los hombres con duración prolongada.

En cuanto a la frecuencia aproximada de la cefalea de manera mensual, los resultados permitieron considerar el hecho de que la mayoría de las mujeres migrañosas reportadas sufren un aumento de sus crisis durante la menstruación. Se relaciona de esta manera con los resultados de la investigación de LarrosaCampo et al (2012) quienes evidenciaron que los cambios hormonales fisiológicos que presentan cíclicamente pueden producir la migraña menstrual.

Respecto a la medicación para combatir el dolor, cabe señalar que los pacientes han probado gran parte de los medicamentos señalados en la teoría; sin embargo, se ha tomado en cuenta aquel medicamento que consume con mayor frecuencia, debido a la condición socioeconómica en la que se sitúa la muestra, y las posibilidades de dotación médica con la que se cuenta en los módulos de salud, resultando prevalencia de utilización del Naproxeno, como antiinflamatorio no esteroideo (AINES). Se ha encontrado con estos resultados que su efectividad ha sido mayor que las opciones terapéuticas de primera línea citadas en los hallazgos de Pérez (2007) y Martínez (2004) en sus respectivas investigaciones, cuando se suministra desde la atención primaria de salud y no se ha instaurado un estatus migrañoso agudo de más de 72 horas de evolución. Cabe destacar que el porcentaje de pacientes que reportó otro tipo de tratamiento en emergencias clínicas incluidos triptanes, benzodiacepinas y clorpromazina, presentaban crisis agudas frecuentes, intensas y de larga duración.

A la exploración física general y neurológica, los resultados fueron habitualmente normales. Sin embargo, se analiza el único elemento con resultados diferentes a la tendencia normal del resto de pasos dentro de la auscultación física, refiriéndose a la cardiopulmonar, específicamente en el aspecto de la tensión arterial, de acuerdo a una posible comorbilidad de las cefaleas; no obstante, este caso está poco estudiado y presente en la literatura, aunque es importante determinar según la investigación de Martin (2004) que un paciente puede ser considerado hipertenso si aparecen varios registros de HTA independientes de las crisis de cefalea. En todo caso, dado el papel de la vasodilatación en la patogenia de la cefalea, principalmente en la migraña, sería razonable que existiera una correlación entre la presión de pulso y la tendencia a la cefalea; aunque ello correspondería a un estudio más especializado respecto a las dos variables.

Los exámenes complementarios no fueron fundamentales en el diagnóstico, excepto en los pocos casos en los cuales se sospecha una lesión orgánica, como tumor cerebral, hemorragia subaracnoidea u otra lesión similar; no obstante, los resultados fueron negativos en los casos que se recomendó tales pruebas. En cuanto a la Prevalencia, se concluye que afecta mayormente a las mujeres, con tipo de migraña sin aura, y edad de 31 a 60 años.

Por otra parte, se evidenció un conjunto de síntomas entre los que se encuentran, además del dolor de cabeza pulsátil, moderado a intenso; sistemáticamente: vómitos, náuseas, sensibilidad a la luz, ruido, presentación de fenómenos visuales, mientras que la pérdida del campo visual no representó porcentajes significativos.

Frente a este escenario, se analiza como aspecto totalmente coincidente con la teoría explicada sobre los síntomas asociados a la migraña donde Goadsby (2012) expone con estricto orden, que los más habituales incluidos en los criterios de diagnóstico, son las náuseas, vómitos y la combinación de fotofobia y fonofobia. Por su puesto, la muestra en investigación refirió como primer síntoma, el dolor según su intensidad, para luego determinar la característica, coincidiendo 
igualmente con los datos anteriormente apreciados en el abordaje diagnóstico, resultante en un dolor moderado a intenso y de carácter pulsátil.

Finalmente, al reconocer los factores de riesgo, según su clasificación, se concluyó que la predisposición ambiental en primer lugar, seguido de los estímulos sensoriales, los cambios de hábitos y ciertos alimentos fueron los factores desencadenantes de episodios de migraña y considerando en gran medida que el calor y las altas temperaturas experimentadas como sensación térmica propio de la región geográfica del Zulia donde la temperatura máxima promedio diaria es más de $34^{\circ} \mathrm{C}$ y la sensación térmica puede sobrepasar los $50^{\circ} \mathrm{C}$, se asocia con las crisis migrañosas de esta población.

Resalta el hecho de que una considerable parte de los pacientes no reconocen algunos de los desencadenantes de su cefalea; siendo importante para identificar de forma oportuna los detonantes del dolor, y lograr un control personal del padecimiento. De esta manera, resulta imperante aportar una serie de recomendaciones de prevención de salud.

Dentro del contexto de resultados en los factores de riesgo, y desde el punto de vista de la medicina comunitaria en atención primaria; fue determinante establecer lineamientos estratégicos de promoción y prevención de salud para evitar los desencadenantes de la migraña, que pueden ser adoptados por los médicos y pacientes de la realidad estudiada y por otros grupos de interés, que presenten situaciones similares a las detectadas en esta investigación.

Como un aporte concluyente, resulta necesario fortalecer tres puntos esenciales bajo un enfoque informativo-terapéutico preventivo, dividiendo lineamientos estratégicos en tres aspectos que engloban los requerimientos en los espacios revisados (Figura 1): Información individualizada al paciente, Jornadas de información comunitaria del tratamiento preventivo y Círculos de calidad de Médicos. En consecuencia, es imprescindible educar al paciente a través de la información adecuada de la patología que le afecta por medio de una consulta de calidad en tiempo y asesoría médica en cuanto a características de la migraña, naturaleza, carácter recurrente, tratamiento preventivo, sintomático y sobre todo los factores de riesgo que predisponen y desencadenan las crisis. Asimismo, es necesario involucrar a la comunidad en una participación protagónica durante jornadas de información, profundizando en el apoyo a los familiares con trastornos migrañosos explicando las diferencias entre tratamiento sintomático y preventivo, tanto farmacológico como no farmacológico.

Figura 1

Portafolio de perspectivas en el enfoque informativoterapéutico preventivo de la migraña

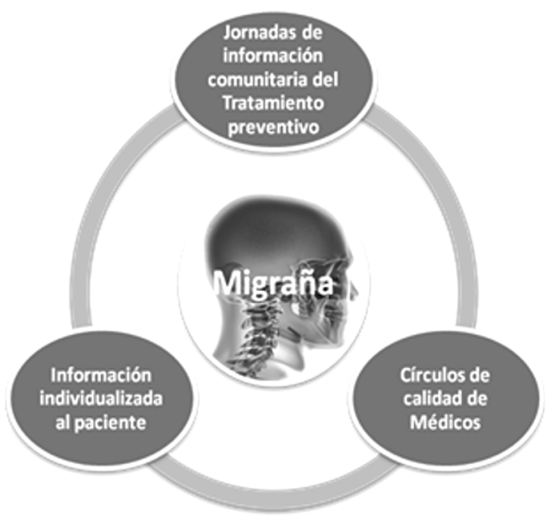

Fuente: Elaboración propia

Finalmente activar círculos de médicos del entorno y de todo personal de salud que desee participar, reuniéndose intencionalmente de modo regular, para identificar casos y llevar a la práctica soluciones oportunas. Ante su elevada prevalencia, este problema de salud debe despertar el interés entre profesionales de Atención Primaria, Neurólogos, profesionales de la salud en general y la comunidad, por cuanto la demanda que sus síntomas provoca, plantea una importante necesidad de mejoramiento en la sociedad.

\section{Declaración de financiamiento y de conflictos de interés:}

El estudio fue financiado por los autores, quienes declaran no tener conflictos de interés.

\section{Correspondencia:}

Elizabeth Salas Acosta

Correo electrónico:

mirelisaeva@gmail.com 
Mireya Salas de González

Correo electrónico:

mireyafine@gmail.com

Melissa González-Salas
Correo electrónico:
melissaagsstar@gmail.com

\section{REFERENCIAS BIBLIOGRÁFICAS}

Artigas-Pallarés J; Grau, R.; Esclusa, F.; Canosa, P y Moltó, E. (1998). "Prevalencia y características de la cefalea y la migraña en la infancia". Rev Neurol; vol.26 (151): 368-371. Recuperado de: https://www.neurologia.com/articulo/98952

Cady RK (2014). "Red flags and comfort signs for ominous secondary headaches". Otolaryngol Clin N Am 2014; Apr 47 (2):289-299. doi: 10.1016/j. otc.2013.10.010.

Chávez, N. (2003). Introducción a la Investigación Educativa. Maracaibo:

Ezpeleta D. (2007). "Diagnóstico diferencial de la cefalea en urgencias" Jano: Medicina y Humanidades 2007; 1662: 31-35.

Goadsby PJ.(2012) "Pathophysiology of migraine". Ann Indian Acad Neurol. 2012 Aug; 15 (Suppl 1): S15-S22. DOI: 10.4103/0972-2327.99993

Hernández, R. Fernández, C. \& Baptista, P. (2010). Metodología de la Investigación. $5^{\mathrm{a}}$ ed. México, DF \& Mc. Graw Hill.

Larrosa-Campo, D.; Ramón-Carbajo, C.; Para-Prieto, M.; Calleja-Puerta, S.; Cernuda-Morollón, E. y Pascual, J. (2012). "La migraña como factor de riesgo vascular”. Rev Neurol 2012; 55 (6): 349358.

Martin V, E. (2004). "Diagnosis and classification of primary headache disorders". In: Standards of care for headache diagnosis and treatment. Chicago (IL): Rev. National Headache Foundation; 2004. p. 4-18.

Martínez, L. (2004) Epidemiología de la migraña en adolescentes. Tesis de postgrado de Neuropediatría. Barquisimeto: Universidad Centro occidental Lisandro Alvarado.

McCandless RT, Arrington CB, Nielsen DC, Bale JF y Minich LLA. (2011). "Patent Foramen Ovale in Children with Migraine Headaches". The Journal Pediatrics. August 2011 Volume 159, Issue 2: 243-247. DOI: https://doi.org/10.1016/j. jpeds.2011.01.062
Organización mundial de la Salud (OMS, 2008). Trastornos neurológicos. Desafíos para la salud pública. Documento en línea. [acceso 15 septiembre 2019]. Disponible en: www1. paho.org/hq/dmdocuments/2008/Trastornos_ Neurologicos.pdf

Oterino, A. y Pascual, J. (2008). "Genética de la migraña" Acta Neurol Colomb 2008. Vol. 24 No. 3 Suplemento (3:1) pp. 34-43. Septiembre 2008 https://www.acnweb.org/acta/2008_24_S3_34. pdf

Padua, J. (2002) Técnicas de Investigación aplicadas a las Ciencias Sociales. México: Fondo de Cultura Económica

Pascual J. (2012). "Migraña crónica: tratamiento". Rev Neurol 2012; 54 (Supl 2): S31-68. https:// psyciencia.com/wp-content/uploads/2012/10/ tratamiento-migrana1.pdf

Pérez, R. (2007) "Migraña. Tratamiento". Revista científica Portales Médicos. Vol II. N¹3, Septiembre, 2007. España

Silberstein, S. (2004). "Migraine pathophysiology and its clinical implications". Cephalalgia. 2004; 24 (Suppl 2): S2-7.

Terwindt, GM.; Ophoff, RA.; Haan, J.; Sandkuijl, LA.; Frants, R. y Ferrari, MD. (1998). "Migraine, ataxia and epilepsy: a challenging spectrum of genetic determined calcium channelopathies". Eur J Hum Genet Jul-Aug 1998;6(4):297-307. doi: 10.1038/ sj.ejhg. 5200206 .

Titus, F; Fraga, C. y Martínez, J. (1992). Clasificación y criterios diagnósticos de las cefaleas, las neuralgias craneales y el dolor facial. Barcelona: MCR.

Uribe García, B. (2008). "Impacto de la migraña". Acta Neurol Colomb Vol. 24 No. 3 Suplemento (3:1) septiembre 2008. https://www.acnweb.org/ acta/2008_24_S3_28.pdf

Vicente, M. (2008). La migraña. Su impacto y repercusión laboral. Tesis de grado publicada. España: Universidad de Valencia. http://hdl. handle.net/10803/10078

Recibido: $17 / 02 / 2021$

Aceptado: 24/05/2021 